\title{
Cooling of isolated neutron stars as a probe of superdense matter physics
}

\author{
Alexander Kaminker*, Alexander Potekhin and Dmitry Yakovlev \\ Ioffe Physical Technical Institute - Politekhnicheskaya 26, 194021 Saint-Petersburg, Russia \\ E-mail: kam@astro.ioffe.ru, palex@astro.ioffe.ru, yak@astro.ioffe.ru
}

\begin{abstract}
We review a current state of cooling theory of isolated neutron stars. The main regulators of neutron star cooling are discussed. We outline the sensitivity of cooling models to equation of state of matter in the neutron star core; the presence or absence of enhanced neutrino emission; superfluidity of baryonic component of matter. A comparison of the cooling theory with observations of thermal emission of isolated neutron stars gives a potentially powerful method to study fundamental properties of superdense matter in neutron star interiors. The prospects of studying neutron star parameters and internal structure are outlined.
\end{abstract}

8th Conference Quark Confinement and the Hadron Spectrum September 1-6, 2008

Mainz. Germany

\footnotetext{
${ }^{*}$ Speaker.
} 


\section{Introduction}

Microscopic theories of superdense matter in neutron star cores are model dependent and give a vast scatter of possible equation of states (EOSs) (e.g., [1, 2]) with different composition of neutron star cores (nucleons, hyperons, pion or kaon condensates, quarks), and different superfluid properties of baryons. The fundamental properties of supranuclear matter cannot be determined on purely theoretical basis, but viable theoretical models can be selected by comparing their predictions with observations of neutron stars. Here we outline some current results obtained from studies of cooling isolated neutron stars (see recent review papers $[3,4]$ for more details and references).

\section{Cooling neutron stars}

Neutron stars have masses $M \sim 1.4 M_{\odot}$ ( $M_{\odot}$ being the Sun's mass). They consist of a thin crust (a layer of mass $\sim 0.01 M$ ) and a massive core (e.g., Ref. [1]). Different EOSs of nuclear matter allow different maximum neutron-star masses between $\sim 1.5 M_{\odot}$ and $2.5 M_{\odot}$. The mass density $\rho$ at the crust-core boundary is $\approx \rho_{0} / 2$, where $\rho_{0} \approx 2.8 \times 10^{14} \mathrm{~g} \mathrm{~cm}^{-3}$ is the density of saturated nuclear matter. The core of a sufficiently massive star can be divided into the outer core [that extends to $\rho \sim(2-3) \rho_{0}$ and consists of neutrons (n), with an admixture of protons (p), electrons (e) and muons, all particles being strongly degenerate] and the inner core that extends to the stellar center [to $\sim(10-20) \rho_{0}$ in most massive stars] and has uncertain composition. Hyperons probably appear in the inner core in addition to nucleons. Other (so-called exotic) models assume the appearance of pion or kaon condensates, quark matter or mixed phases (as reviewed, e.g., in Ref. [1]). Nucleons, hyperons and quarks can be in superfluid state.

Neutron stars are born in supernova explosions with internal temperature $T \sim 10^{11} \mathrm{~K}$ and gradually cool down. In $\sim 30 \mathrm{~s}$ after the explosion a star becomes transparent for neutrinos (generated in its interiors) and transforms from a proto-neutron star to an ordinary neutron star (as reviewed, e.g., in [5]). At the later neutrino-transparent stage the star cools via neutrino emission from its interior and via heat transport to the surface and photon emission from there. A distant observer detects the "apparent" effective temperature $T_{\mathrm{s}}^{\infty}=T_{\mathrm{s}} \sqrt{1-r_{\mathrm{g}} / R}$, where $T_{\mathrm{s}}$ is the local effective surface temperature and $r_{\mathrm{g}}=2 G M / c^{2}$ is the Schwarzschild radius. The theory gives cooling curves, $T_{\mathrm{S}}^{\infty}(t)$, and predicts three main cooling stages.

The initial stage of internal thermal relaxation lasts $\sim 10-50 \mathrm{yr}$. At this stage, the crust stays hotter than the core because of intense neutrino cooling of the core $\left(T_{\mathrm{s}}\right.$ is then insensitive to the physics of the core being determined by the physics of the crust $[6,7])$. The next stage of neutrino cooling with isothermal interior lasts $\sim 10^{5}-10^{6}$ yr. At this stage the neutrino luminosity $L_{v}$ remains much larger than the thermal photon luminosity $L_{\mathrm{S}}$ of the star and regulates the cooling. At the later photon cooling stage the neutrino emission dies out $\left(L_{v} \ll L_{\mathrm{S}}\right)$ and the cooling is governed by photon thermal emission from the surface. At the two latter stages $T_{\mathrm{s}}$ is coupled to the internal temperature via thermal conduction in a thin outer heat-blanketing envelope.

\section{Neutrino emission mechanisms}

The neutrino processes in the core can be divided into slow and fast ones (e.g., [3, 8-11]). In non-superfluid dense matter the emissivity of slow and fast neutrino processes can be written 
as $Q_{\text {slow }}=Q_{\mathrm{s}} T_{9}^{8}$ and $Q_{\text {fast }}=Q_{\mathrm{f}} T_{9}^{6}$, where $T_{9}=T /\left(10^{9} \mathrm{~K}\right)$, while $Q_{\mathrm{s}}$ and $Q_{\mathrm{f}}$ (hereafter measured in $\left.\mathrm{erg} \mathrm{cm} \mathrm{cm}^{-3} \mathrm{~s}^{-1}\right)$ are slowly varying functions of $\rho$ listed in Tables 3 and 4 of Ref. [3]. Slow (weak) neutrino processes in nucleon matter of the outer core $\left(\rho \lesssim 2 \rho_{0}\right)$ are modified Urca process $\left(\mathrm{nN} \rightarrow \mathrm{pNe} \bar{v}, \mathrm{pNe} \rightarrow \mathrm{nN} v ; \mathrm{Q}_{\mathrm{s}} \sim 10^{20}-3 \times 10^{21}\right)$ and nucleon-nucleon bremsstrahlung (NN $\rightarrow \mathrm{NN} v \bar{v} ; \mathrm{Q}_{\mathrm{s}} \sim 10^{19}-10^{20}$ ), where $\mathrm{N}$ is a nucleon ( $\mathrm{n}$ or $\mathrm{p}$ ). The modified Urca process differs from its direct Urca progenitor, described below, by an additional nucleon $\mathrm{N}$ that is required to satisfy momentum conservation of reacting particles. All reactions involving electrons can involve muons instead.

At higher $\rho$, in the inner core, neutrino emission can be strongly enhanced by new processes (Table 3 in [3]). Such enhancement depends on the EOS and composition of superdense matter that is most important for the cooling problem. The strongest enhancement is provided by the direct Urca process [12, 13] in nucleon (or nucleon-hyperon) matter $\left(\mathrm{n} \rightarrow \mathrm{pe} \bar{v}, \mathrm{pe} \rightarrow \mathrm{n} v ; \mathrm{Q}_{\mathrm{f}} \sim 10^{26}-3 \times\right.$ $10^{27}$ ). It is allowed in the matter with proton fraction $\gtrsim 11-13 \%$. If it is forbidden but the matter contains pion condensate, neutrino emission is enhanced by direct Urca-type reactions involving quasi-nucleons $\widetilde{\mathrm{N}}$ (superpositions of $\mathrm{n}$ and $\mathrm{p}$ ) in pion-condensed matter $\left(\widetilde{\mathrm{N}} \rightarrow \widetilde{\mathrm{Ne}} \bar{v}, \widetilde{\mathrm{Ne}} \rightarrow \widetilde{\mathrm{N}} v ; \mathrm{Q}_{\mathrm{f}} \sim\right.$ $\left.10^{23}-10^{26}\right)$. If pion condensate is absent, but kaon condensate available, neutrino emission can still be enhanced (though weaker) by direct Urca-like processes involving baryonic quasiparticles $\widetilde{\mathrm{B}}$ in kaon condensed matter $\left(\mathrm{Q}_{\mathrm{f}} \sim 10^{23}-10^{24}\right)$. Nearly the same enhancement is expected due to the direct Urca process involving $\mathrm{d}$ and $\mathrm{u}$ quarks in quark matter $(\mathrm{d} \rightarrow \mathrm{ue} \bar{v}$, ue $\rightarrow \mathrm{d} v)$. The neutrino processes in the inner neutron star core can enhance the neutrino emission by $2-7$ orders of magnitude and lead to fast cooling.

Nucleons, hyperons, and quarks in dense matter can be in superfluid state (e.g., [14], also see $[3,9,11]$ for references). Their Cooper pairing occurs due to an attractive component of their interaction. Microscopic calculations of superfluid critical temperatures $T_{\mathrm{c}}(\rho)$ of different particles are very model dependent. Baryon superfluidity affects neutrino emission and heat capacity of dense matter.

\section{Slow and fast neutron star cooling}

The majority of proposed cooling scenarios predict that (see, e.g., $[15,3,11])$ :

(1) Low-mass neutron stars (no enhanced neutrino emission in the core) undergo slow cooling;

(2) High-mass stars cool faster via enhanced neutrino emission from their inner core;

(3) There is a family of medium-mass stars whose cooling is intermediate.

In particular, proton superfluidity can be strong in the outer core and suppress the modified Urca process. If so, the slow cooling of low-mass stars is determined by weaker neutrino bremsstrahlung process due to nn-collisions. It is sufficient to lower the neutrino luminosity by a factor of $\sim 30-100$ below the modified Urca level to explain observations of stars hottest for their age. In addition, proton superfluidity can penetrate into the inner core and suppresses the direct Urca process there (within the penetration depth), realizing a smooth transition from slowly to rapidly cooling neutron stars with the growth of $M$.

To analyze the level of neutrino emission in high-mass stars, let us consider (Fig. 1 from [3]) three typical levels appropriate to the direct Urca process in nucleon/hyperon matter, pion- 

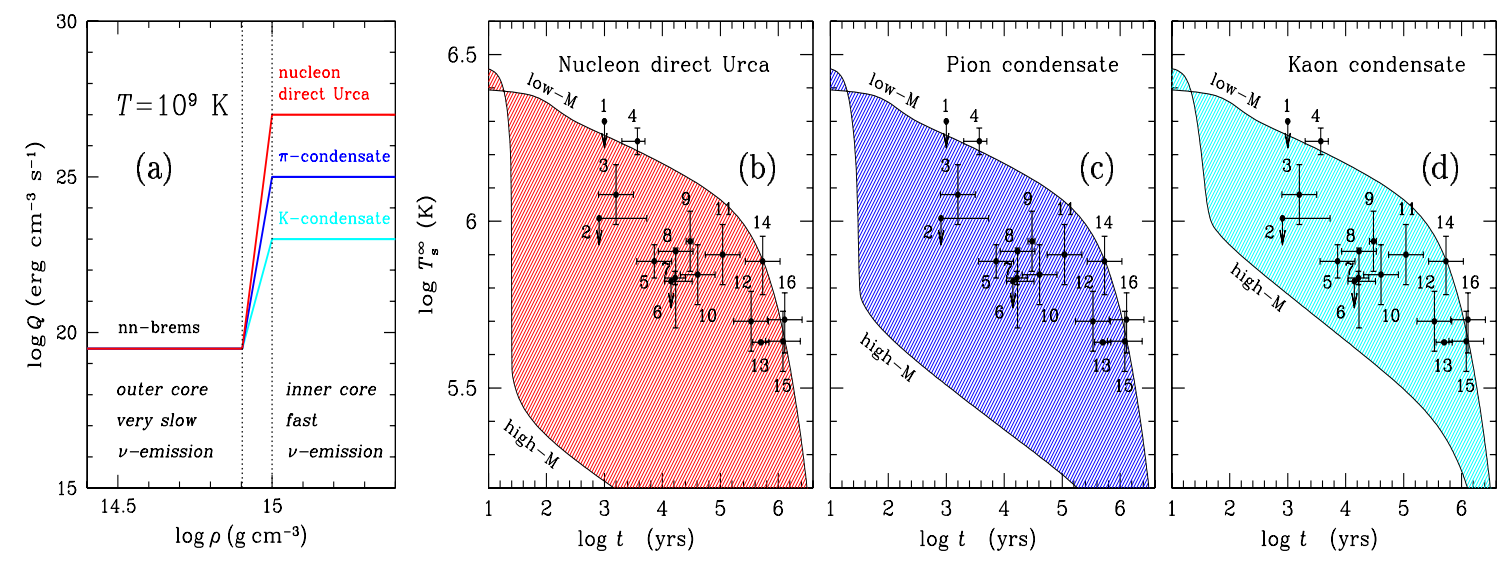

Figure 1: (a) Schematic density dependence of the neutrino emissivity in a neutron star core at $T=10^{9} \mathrm{~K}$ assuming slow neutrino emission in the outer core and three scenarios of fast emission in the inner core. (b), (c), and (d): Ranges of $T_{\mathrm{s}}^{\infty}$ (hatched) for the three types of fast emission compared with observations (see text for details). Also shown are observational limits on surface temperatures of 16 cooling neutron stars [11]: Crab pulsar (1); PSR J0205+6449 (2); PSR J1119-6127 (3); RX J0822-4300 (4); PSR J1357-6429 (5); RX J0007.0+7303 (6); Vela pulsar (7); PSR B1706-44 (8); PSR J0538+2817 (9); PSR B2334+61 (10); PSR B0656+14 (11); Geminga (12); RX J1856.4-3754 (13); PSR B1055-52 (14); PSR J2043+2740 (15); RX J0720.4-3125 (16).

condensed matter and kaon-condensed matter. A schematic picture of the neutrino emissivity profiles through the stellar core for these scenarios is presented in Fig. 1a.

Figure $1 \mathrm{~b}$ shows the (hatched) ranges of $T_{\mathrm{s}}^{\infty}$ consistent with the nucleon direct Urca process. The upper curve corresponds to low-mass stars which cool via nn-bremsstrahlung neutrino emission. The lower curve shows fast cooling of a maximum-mass star with the direct Urca process open in the inner core. Any value of $T_{\mathrm{s}}^{\infty}$ between these two curves can be explained by a cooling of some intermediate-mass star. This scenario predicts the existence of very cold neutron stars.

Figure 1c refers to the scenario with pion-condensation. The upper curve is the same as in Fig. 1b, but the lower curve goes essentially higher (neutrino emission due to pion condensation is weaker). This scenario also predicts the existence of cold stars, but not so cold as in Fig. 1a. Fig. $1 \mathrm{~d}$ is similar to Fig. 1c but with kaons instead of the pions. High-mass stars cool slower than in the previous scenarios; a population of stars only slightly colder than the observed ones is predicted.

Thus, all three scenarios are currently consistent with the observations, but predict different families of cold stars.

Our main conclusions are:

1. The theory of cooling neutron stars of ages $10^{2}-10^{6} \mathrm{yr}$ mostly tests the neutrino emission properties of the star's core.

2. Neutrino emission in the core of a low-mass star is a factor of 30-100 lower than the modified Urca emission in a non-superfluid star.

3. Neutrino emission in the inner core of a massive star is at least a factor of 30-100 higher than the modified Urca emission. It can be enhanced by the direct Urca process in nucleon/hyperon inner core or by the presence of pion or kaon condensate, or quark matter.

4. The scenario with open direct Urca process in nucleon/hyperon matter predicts the existence of 
massive isolated neutron stars which are much colder than those observed now.

5. A discovery of cold cooling neutron stars would be crucial to constrain the level of enhanced neutrino emission and, as consequence, the EOS of matter in the inner core.

6. A transition from slow neutrino emission in the outer core to enhanced emission in the inner core has to be smooth. A firm measurement of masses of cooling stars would help to impose constraints on parameters of this transition.

7. New observations and reliable practical theories of dense matter are vitally important to tune the cooling theory as a probe of physical properties of neutron star interiors.

\section{Acknowledgments}

This work was supported by the grants of RFBR (08-02-00837) and FASI-Rosnauka (NSh 2600.2008.2).

\section{References}

[1] P. Haensel, A.Y. Potekhin and D.G. Yakovlev, Neutron Stars. 1. Equation of State and Structure, Springer, New York, 2007.

[2] J.M. Lattimer and M. Prakash, Neutron star observations: Prognosis for equation of state constraints, Phys. Rep., 442 (2007) 109-165.

[3] D. G. Yakovlev and C. J. Pethick, Neutron star cooling, Annu. Rev. Astron. Astrophys., 42 (2004) 169-210.

[4] D. Page, U. Geppert and F. Weber, The cooling of compact stars, Nucl. Phys. A, 777 (2006) 497-530.

[5] M. Prakash, J. Lattimer, J. A. Pons, A. W. Steiner and S. Reddy, Evolution of a neutron star from its birth to old age, Lecture Notes in Physics, 578 (2001) 364-423.

[6] J. M. Lattimer, K. A. Van Riper, M. Prakash and M. Prakash, Rapid cooling and the structure of neutron stars, Astrophys. J., 425 (1994) 802-813.

[7] O. Y. Gnedin, D. G. Yakovlev and A. Y. Potekhin, Thermal relaxation in young neutron stars, Mon. Not. Roy. Astron. Soc., 324 (2001) 725-736.

[8] C. J. Pethick, Cooling of neutron stars Rev. Mod. Phys., 64 (1992) 1133-1140.

[9] D. G. Yakovlev, K. P. Levenfi sh and Yu. A. Shibanov, Cooling of neutron stars and superfluidity in their cores, Physics - Uspekhi, 42 (1999) 737-778.

[10] D. G. Yakovlev, A. D. Kaminker, O. Y. Gnedin and P. Haensel, Neutrino emission from neutron stars, Phys. Rep., 354 (2001) 1-155.

[11] D. G. Yakovlev, O. Y. Gnedin, A. D. Kaminker and A. Y. Potekhin, Theory of cooling neutron stars versus observations, AIP Conf. Proc., 983 (2008) 379-387.

[12] J. M. Lattimer, C. J. Pethick, M. Prakash and P. Haensel, Direct URCA process in neutron stars, Phys. Rev. Lett., 66 (1991) 2701-2704.

[13] M. Prakash, M. Prakash, J. M. Lattimer and C. J. Pethick, Rapid cooling of neutron stars by hyperons and $\Delta$-isobars, Astrophys. J., 390 (1992) L77-L80.

[14] U. Lombardo and H.-J. Schulze, "Superfluidity in neutron star matter" in Physics of Neutron Star Interiors, edited by D. Blaschke, N. Glendenning, and A. Sedrakian (Springer, Berlin 2001) 30-53.

[15] A. D. Kaminker, D. G. Yakovlev and O. Y. Gnedin, Three types of cooling superfluid neutron stars: Theory and observations, Astron. Astrophys., 383 (2002) 1076-1087. 